\title{
Protocol
}

\section{Scoring and Analyzing Aggression in Drosophila}

\author{
Sarah J. Certel and Edward A. Kravitz
}

Aggression is an innate behavior that has likely evolved in the framework of defending or obtaining resources. This complex social behavior is influenced by genetic, hormonal, and environmental factors. In many organisms, aggression is critical to survival, but the ability to control and suppress aggression in distinct contexts also is necessary. Invertebrate organisms, with their relatively simple nervous systems and a multiplicity of powerful tools available to examine their often elaborate and complex behavioral displays, have become increasingly valuable models for investigating the genetic and systems biological roots of social behavior. In this protocol, we outline methods for analyzing aggression in Drosophila: The design encompasses eco-ethological constraints that emphasize an understanding of normal aggression. The details include steps for constructing a fight arena, isolating and painting flies, introducing flies to an arena, and videotaping and scoring fights. These experimental protocols are in current use to identify candidate genes important in aggression and to elaborate the neuronal circuitry underlying the display of aggression and other social behaviors.

\section{MATERIALS}

It is essential that you consult the appropriate Material Safety Data Sheets and your institution's Environmental Health and Safety Office for proper handling of equipment and hazardous materials used in this protocol.

Reagents

Acrylic paint

Agarose (2\%; molecular biology grade)

Prepare by mixing the agarose with deioinized water and microwaving until it fully dissolves.

$\mathrm{CO}_{2}$ for anesthesia

Drosophila pupae and adult flies (e.g., 3-5-d old)

Keep all flies (stock and cross vials, isolation tubes with pupae) in an environmentally controlled 12-h light/ 12-h dark cycle incubator at the desired temperature (e.g., $25^{\circ} \mathrm{C}$ ). Maintain eclosed, aging flies in the same incubator to control their activity periods.

Dry active yeast

Fly food, standard

Equipment

Black filter paper circles $(8.5-\mathrm{cm})$ with $2-\mathrm{cm}$ hole cut in center

Camera (Sony DCR-HC62 [or model with good optics])

Computer (Mac)

Adapted from Drosophila Neurobiology (ed. Zhang et al.). CSHL Press, Cold Spring Harbor, NY, USA, 2010.

(C) 2012 Cold Spring Harbor Laboratory Press

Cite this article as Cold Spring Harbor Protoc; 2012; doi:10.1101/pdb.prot068130 
S.J. Certel and E.A. Kravitz

Diamond glass cutter

Glue (Dow Corning DAP 100\% silicone household adhesive for ceramics and china or Devcon High Strength 5 Minute Epoxy)

Incubator, environmentally controlled 12 -h light/12-h dark cycle, preset to the desired temperature (e.g., $\left.25^{\circ} \mathrm{C}\right)$

Incubator, high-humidity environment $(45 \%-55 \%)$, preset to $25^{\circ} \mathrm{C}$

Isolation vials, borosilicate glass culture tubes $(16 \times 100 \mathrm{~mm})$ (VWR International 47729-576)

Laboratory label tape, colored

Light source

Microscope slides, plain (Fisher Scientific)

Mortar and pestle, small

Pasteur pipette

Petri dishes $(100 \times 15 \mathrm{~mm}$, polystyrene disposable, sterile) (VWR International)

Ruler

Scintillation vial caps $(15 \times 10 \mathrm{~mm})($ Kimble $)$

Straight dissecting needle with birch handle

Toothpicks, wooden

$\stackrel{\circ}{\circ}$ METHOD

A complete video presentation of this protocol can be found at the JoVE online journal (see Mundiyanapurath et al. 2007).

Assembling the Chamber Walls

See Figure 1.

1. Take two plain glass slides, mark the middle at the two edges, and, using a straight-edged ruler as a guide, score the glass with the diamond cutter. Slide the diamond cutter back and forth a few times, generating a scraping noise.

2. Gently apply pressure to the scored line to break the slide into two halves. Face the scored side away from you for safety.

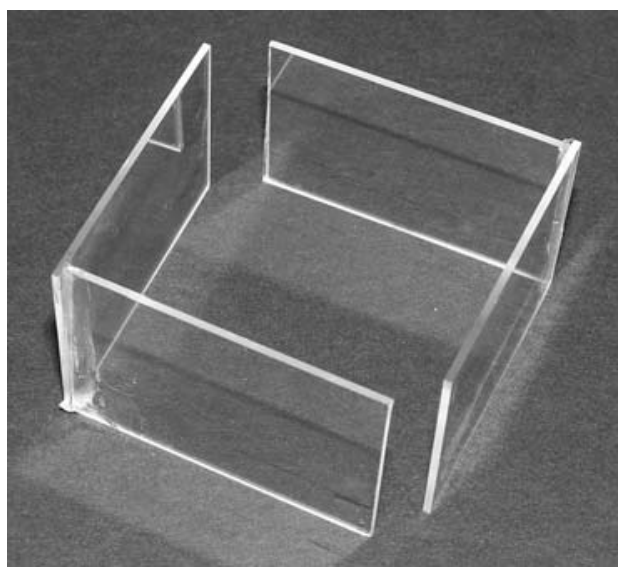

FIGURE 1. Two sides of the fighting chamber glued together. The two halves of a marked, broken standard microscope slide were glued together at right angles. 


\section{Making the Food Cup}

3. Generously apply the glass adhesive along an edge of one piece of the broken slide. Then align and firmly press the second slide piece onto the adhesive. Place onto a flat surface and allow to set for at least $1 \mathrm{~h}$.

4. After $1 \mathrm{~h}$, repeat the application of the adhesive step to the other edges and form a square chamber with the second two-slide piece. Allow the glue to harden overnight.

\section{Placing the Walls in a Petri Dish}

See Figure 2.

5. Fill the top of a Petri dish to a depth of at least $5 \mathrm{~mm}$ with a heated solution of $2 \%$ agarose.

6. Wait a few minutes, but while the agarose is still molten, place the walls of the chamber in the center of the dish.

7. Using a flame-heated straight dissecting needle, make a set of small holes $(>1 \mathrm{~mm})$ in the center of the bottom of the Petri dish for ventilation, and make a larger hole ( $\sim 4 \mathrm{~mm}$ diameter) slightly to the side for introducing flies to the chamber. Place a piece of removable label tape over the larger hole.

\section{See Figures 2 and 3}

8. Heat a vial of fly food to the point at which the food is melted. Use a Pasteur pipette to transfer the food to a scintillation vial cap, filling it to form a flat surface at the top. Be careful to avoid air bubbles and allow the food to cool.

9. Make fresh yeast paste by grinding a small amount of dry yeast in a mortar and pestle and adding a few drops of water. Add more yeast paste or water until the consistency is thick enough to allow a small visible drop to be picked up by a toothpick and applied to the center of the scintillation vial cap food surface.

10. Place the vial cap containing food in the center of the chamber and place the bottom of the Petri dish containing the small holes, inverted so that the sides are up and not obscuring the view, on top of the chamber.

11. Introduce two 3-5-d-old flies by aspiration into the chamber through the larger hole in the top, cover that hole with tape, and place a piece of black filter paper with a $2-\mathrm{cm}$ hole cut in it into the top dish.

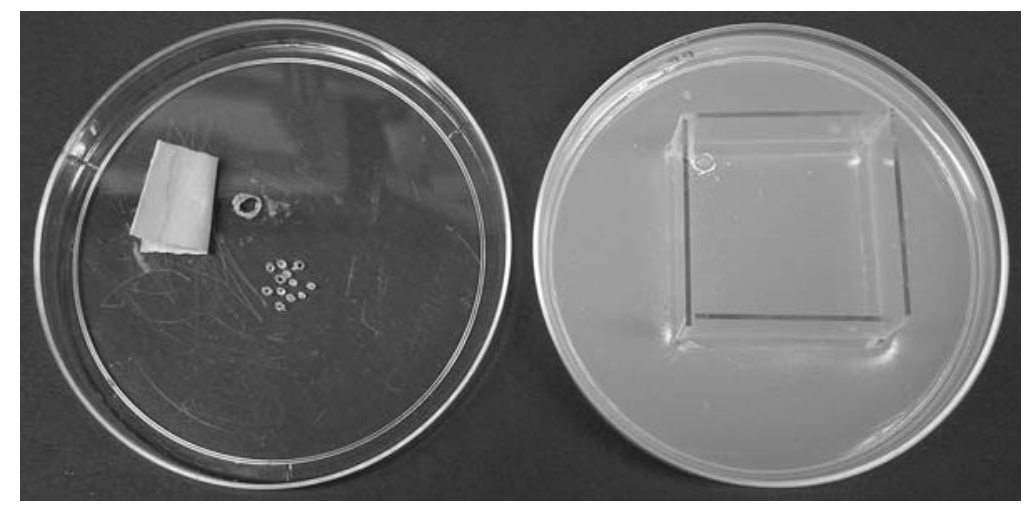

FIGURE 2. Top view of the two fighting chamber parts. The glass box has been placed into a $1 \%$ agarose solution. Ventilation holes and a single larger aspiration hole have been made in the cover. 
S.J. Certel and E.A. Kravitz
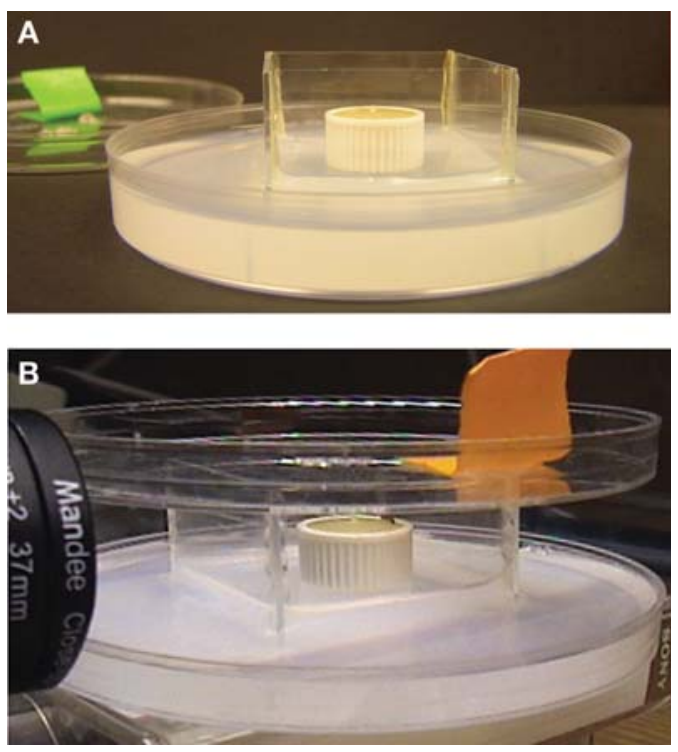

FIGURE 3. Side view of a finished chamber showing placement of the scintillation vial/food cup $(A)$ and videotaping angle $(B)$.

12. Place a light source above the chamber, far enough away that it will not heat the chamber, and positioned to effectively illuminate the entire food surface.

13. Move the entire assembly into a high-humidity environment $(45 \%-55 \%)$ at a temperature of $25^{\circ} \mathrm{C}$.

\section{Social Isolation and Painting}

Select flies maintained in an environmentally controlled 12-h light/12-h dark cycle incubator at the desired temperature (e.g., $25^{\circ} \mathrm{C}$ ). Begin fights within the first hour after the onset of the light cycle to maximize activity and to reduce possible circadian rhythm effects.

14. Prepare isolation vials by adding $1.5 \mathrm{~mL}$ of boiled fly food to individual glass tubes. Allow the food to solidify.

15. Gently remove a late-stage male or female pupa from the cross or stock vial and place it on the side of the isolation vial.

16. Return isolation vials plus pupae to the light/dark cycle incubator.

17. Paint eclosed flies for individual identification as follows. (Perform this at least $24 \mathrm{~h}$ before fighting to eliminate any possible effects of the anesthesia.)

i. Anesthetize the flies under $\mathrm{CO}_{2}$.

ii. Place a tiny dab of acrylic paint on the thorax with a toothpick. Avoid contact of paint with head, wings, or abdomen.

iii. Allow the paint to dry briefly, then gently transfer the fly back into the isolation vial.

\section{Videotaping and Behavioral Analysis}

As shown in Figure 3B, video cameras are positioned to record an oblique view of the fighting territory and the pair of flies.

18. Record the fights (usually for up to $1.5 \mathrm{~h}$ ), beginning the video recording when flies are first placed in the arena.

i. To view encounters, attach the camera to a Mac computer.

ii. Open the iMovie program and begin playing the tape. 
iii. Record the start time, the time when both flies are first together on the food surface, and the time of the first encounter (meeting between the flies when they start to interact and are within one body length of each other).

iv. Score the time period of latency to the first encounter.

19. Create and observe movie clips of the individual encounters. For examples of movie clips, see Movies 1, 2, and 3 at http://cshprotocols.cshlp.org.

i. Use the space bar on the iMovie program (see iMovie Help) to generate clips of individual encounters.

ii. Examine each clip or encounter either at a single frame level or at reduced speeds, and observe the behavioral patterns during each encounter. Score the encounters and behavioral patterns.

iii. If desired, score also the encounter duration, time between encounters, as well as any other information of interest, either from the original tapes or from the clipped encounters.

20. Enter the data from individual fights into Excel spreadsheets. Gather and record also any other pertinent data, including the genotypes of the paired animals, the calendar date, numbers of encounters in the fight, the patterns observed, and whether or not a hierarchical relationship was established.

\section{DISCUSSION}

Aggression in fruit flies was not well known until the publication of recent papers demonstrating that (a) fighting behavior could be observed between pairs of male and pairs of female flies in simplified fight arenas; (b) some behavioral patterns seen in male and female fights were similar whereas others were sex selective; (c) males formed hierarchical relationships, whereas females did not; (d) quantitative analyses of the behavior could be performed using traditional methods of behavioral analysis to reveal the structure of average fights including the construction of ethograms, the generation of transition matrices, and the calculation of first-order Markov chains (Chen et al. 2002; Nilsen et al. 2004); and (e) genes could be identified that were selectively associated with aggression (Dierick and Greenspan 2006; Edwards et al. 2006). Drosophila fights resemble those examined in other species in that they are broken up into brief encounters (meetings between the flies) where flies come together, interact for a period of time, and then break apart again. During each encounter, a variety of behavioral patterns are seen: These show statistically varying transitions between them. In male fights, the patterns include approach, fencing, wing threats (of $>2 \mathrm{sec}$ duration), lunging, holding, boxing, tussling, chasing, and retreat. In female fights, approach, fencing, and brief wing threats that resemble the patterns seen in males are observed. At the mid-intensity level, however, instead of lunging, females shove each other and head butt the opponent in competition for resources. These sex-specific aggressive patterns are robust and used in complex social situations involving multiple male and female flies as reported in previous studies (Dow and von Schilcher 1975; Hoffmann 1987a,b; Ueda and Kidokoro 2002; Dierick and Greenspan 2006). Studies from the Hoffmann laboratory (Hoffmann 1987a), in particular, carefully characterized and identified many of the behavioral patterns that can also be observed in the one-on-one fight situation described here.

\section{Designing an Experimental Arena for the Study of Behavior}

Because aggression is used in the wild to defend or to obtain resources, it seems reasonable to include resources in the design of laboratory fight arenas. Thus, conditions can be established that resemble behavioral situations that animals normally encounter in the wild (Blanchard et al. 2003; Branchi and Ricceri 2004; Crabbe and Morris 2004). We therefore considered two key areas in designing our experimental paradigm: First, we attempted to include ethological constraints within our design (e.g., in the wild, losers commonly leave a resource or territory after social defeat; therefore space 
for retreat was included in our arenas); second, it is important to record as much of the behavior shown by the animals as possible for use in later quantitative analyses. Both considerations are essential in attempting to relate molecular findings to normal and abnormal behavioral observations.

Designing behavioral paradigms that include ethological considerations can be difficult: It is necessary to be able to constantly and consistently observe the behavior and, at the same time, provide laboratory conditions that resemble the real world. Fruit flies are, after all, real animals that respond to their environment as strongly as they do to the presence of another animal. In the paradigm outlined here, behavior is viewed with video cameras positioned at an oblique angle to the side of the fight arena. This angle allows accurate video images of the complex patterns and fight sequences shown by both animals viewed in three-dimensional space.

Routine analysis of the behavior involves tallying all of the behavioral patterns seen in individual encounters throughout fights. Other events that can be scored include latency to initiate fighting, number of encounters, winners and losers of individual encounters, and whether or not a hierarchical relationship is established. It is also important to consider the pairings of flies for fights. For example, pairing mutant and control flies may offer a relevant ethological situation, addressing questions of whether the mutants can win fights against wild-type opponents. However, the outcome of this particular pairing may be that mutant flies continually retreat from control flies, thereby always losing fights. To observe what aggressive responses a mutant fly is actually capable of performing, two mutant flies can be paired. The analysis of both types of pairings might provide different but useful data in understanding how a particular gene functions in a behavioral context. Finally, females fight for resources in same-sex pairing too. Thus studies that use genetic or engineered mutants and show interesting behavioral phenotypes in male flies also should be examined with pairs of female flies.

\section{Observing and Analyzing Aggressive Behavior}

For a behavior to evolve by natural selection, the reproductive benefits must exceed the reproductive costs of displaying that behavior. During aggression, the costs include risk of injury and death in combat between animals possessing dangerous weapons. As a consequence, sequences of interactions at different levels of intensity have evolved within most species that can lead to conflict resolution without escalation to all-out fighting behavior (Archer 1988; Wingfield et al. 1993). In defining aggressive status, investigators often ignore intermediate steps and score only the terminal phases of agonistic conflicts, the escalated fights. There is much to be gained, however, in analyses that include observation of all phases of the agonistic meetings between pairs of organisms. Included would be information on what triggers the aggression, the motivation of animals to fight, the progression of fights, changes in the motor patterns used by animals during fights, and, ultimately, answering the question of whether the usage of particular patterns defines how a conflict is resolved. In the fight chambers we use, for example, an ultimate winner might lunge more than 100 times before an ultimate loser retreats from the food surface. The key to the outcome of a fight of this type might not be increased aggressiveness by the ultimate winner, but the refusal of the ultimate loser to back down.

Use of the experimental paradigm described here and subjecting the gathered data to a comprehensive analysis of the patterns of behavior seen during fights is labor intensive. For high-throughput screening of mutants, it may not be practical to perform primary screens in this way. In these situations, it might be better to use smaller size chambers in a primary screen, followed by examination of any interesting mutants using a secondary screen allowing a thorough behavioral analysis to be performed. If one is interested in aggression as it relates to the normal usage of this behavior in the wild, whatever chamber is used should contain a defined territory and/or resources that animals might ordinarily be willing to compete over. Key fight components to examine in simplified scoring protocols might include latency to first encounter, latency to first lunge, and numbers of lunges performed by ultimate winners and losers. It is also important to ask whether a hierarchical relationship has been established within the time window of the fight. Additional parameters that can be scored in male fights include whether high-intensity aggressive components like boxing and tussling are seen 
and/or whether courtship components like wing extensions and abdomen bending are observed during fights.

As Drosophila geneticists, we understand that observing divergence from normality in the case of traditional or engineered mutants offers insights into the normal function of genes, neurons, and neuronal circuits. In extrapolating these techniques to the study of behavior in Drosophila, it is important to remember that the behavioral patterns observed during agonistic encounters are part of a complex repertoire that includes many behavioral patterns, each one of which bears a certain statistical likelihood of transitioning to another. Ignoring the complexity of the social situation may lead to false conclusions as to the consequences of created mutations. On the other hand, with careful examination of the behavior, valuable information can be gained toward understanding what aspects of aggression are fundamental to fights in all species of animals and which are selective for the species under examination.

\section{ACKNOWLEDGMENTS}

We thank members of the Kravitz Laboratory for many helpful discussions. This work has been supported by research grants from the National Institute of General Medical Sciences (GM074675 and GM067645 to E.A.K) and the National Science Foundation (IDS-075165 to E.A.K.).

\section{REFERENCES}

Archer J. 1988. The behavioural biology of aggression. Cambridge University Press, New York.

Blanchard RJ, Wall PM, Blanchard DC. 2003. Problems in the study of rodent aggression. Horm Behav 44: 161-170.

Branchi I, Ricceri L. 2004. Refining learning and memory assessment in laboratory rodents. An ethological perspective. Ann Ist Super Sanita 40: 231-236.

Chen S, Lee AY, Bowens NM, Huber R, Kravitz EA. 2002. Fighting fruit flies: A model system for the study of aggression. Proc Natl Acad Sci 99: 5664-5668.

Crabbe JC, Morris RG. 2004. Festina lente: Late-night thoughts on highthroughput screening of mouse behavior. Nat Neurosci 7: 1175-1179.

Dierick HA, Greenspan RJ. 2006. Molecular analysis of flies selected for aggressive behavior. Nat Genet 38: 1023-1031.

Dow MA, von Schilcher F. 1975. Aggression and mating success in Drosophila melanogaster. Nature 254: 511-512.

Edwards AC, Rollmann SM, Morgan TJ, Mackay TF. 2006. Quantitative genomics of aggressive behavior in Drosophila melanogaster. PLoS Genet 2: e154. doi: 10.1371/journal.pgen.0020154.
Hoffmann AA. 1987a. A laboratory study of male territoriality in the sibling species Drosophila melanogaster and D simulans. Anim Behav 35: 807-818.

Hoffmann AA. 1987b. Territorial encounters between Drosophila males of different sizes. Anim Behav 35: 1899-1901.

Mundiyanapurath S, Certel S, Kravitz EA. 2007. Studying aggression in Drosophila (fruit flies). J Vis Exp 25: 155.

Nilsen SP, Chan YB, Huber R, Kravitz EA. 2004. Gender-selective patterns of aggressive behavior in Drosophila melanogaster. Proc Natl Acad Sci 101: 12342-12347.

Ueda A, Kidokoro Y. 2002. Aggressive behaviours of female Drosophila melanogaster are influenced by their social experience and food resources. Physiol Entomol 27: 21-28.

Wingfield JC, Doak D, Hahn TP. 1993. Integration of environmental cues regulating transitions of physiological state, morphology and behavior. Academic, New York. 


\section{Scoring and Analyzing Aggression in Drosophila}

Sarah J. Certel and Edward A. Kravitz

Cold Spring Harb Protoc; doi: 10.1101/pdb.prot068130

\begin{tabular}{rc}
\hline $\begin{array}{r}\text { Email Alerting } \\
\text { Service }\end{array}$ & Receive free email alerts when new articles cite this article - click here. \\
\hline $\begin{array}{c}\text { Subject } \\
\text { Categories }\end{array}$ & $\begin{array}{c}\text { Browse articles on similar topics from Cold Spring Harbor Protocols. } \\
\text { Behavioral Assays (99 articles) } \\
\text { Drosophila (272 articles) } \\
\text { Image Analysis (124 articles) } \\
\text { In Vivo Imaging, general (168 articles) } \\
\text { Video Imaging / Time Lapse Imaging (171 articles) }\end{array}$ \\
\hline
\end{tabular}

